\title{
Las orquestas comunitarias en Baja California: la experiencia del programa Redes 2025
}

\author{
Emiliano López Guadarrama*
}

La naturaleza del niño encuentra en la música los elementos necesarios para su desarrollo tanto como la naturaleza de la música encuentra en el niño su razón de vida y emancipación.

PABLO TORRES PARÉS

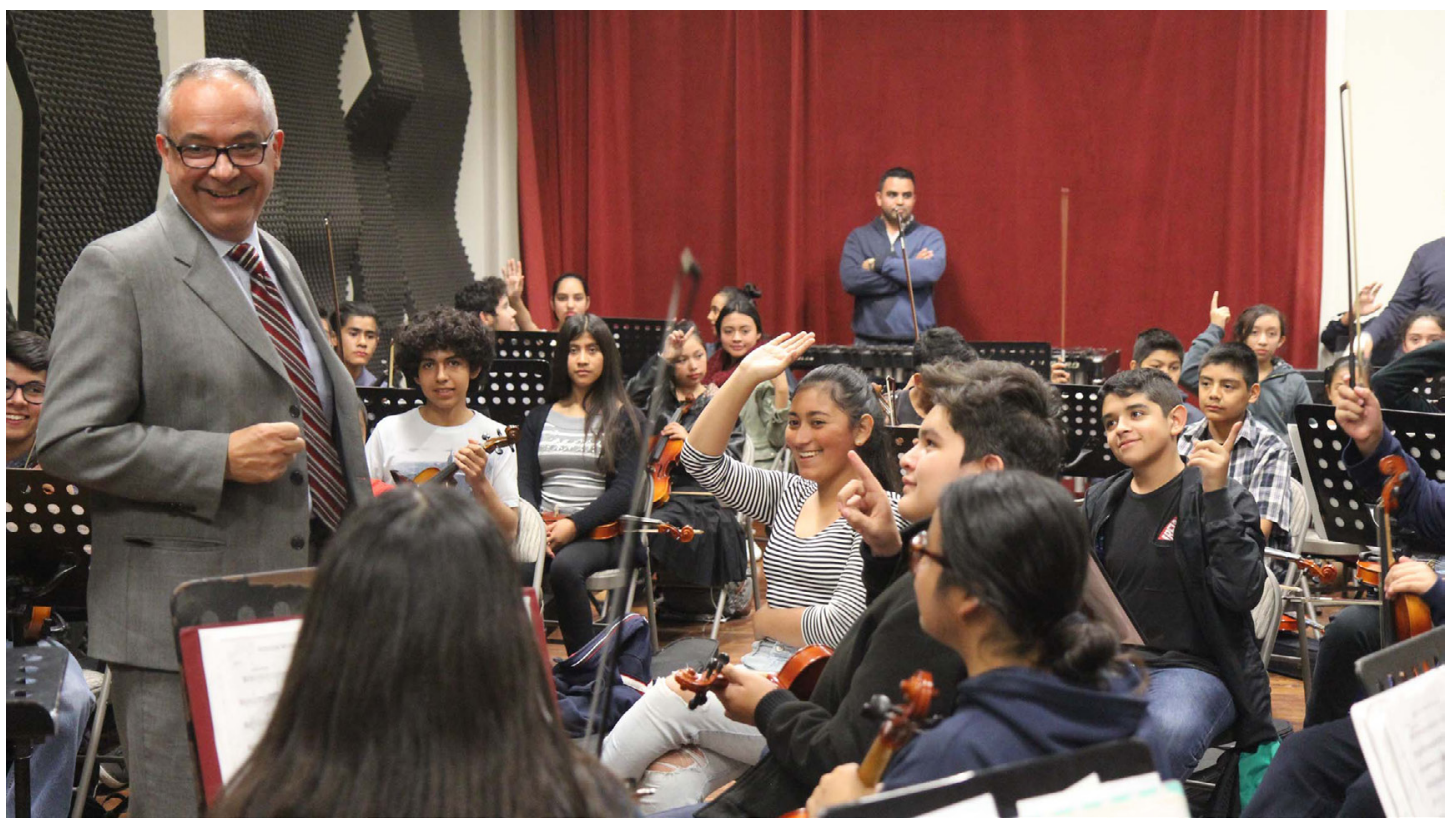

Cuando uno es músico es muy difícil decir dónde y cómo comenzó todo. Lo cierto es que todo niño nace con un potencial inmenso de interacción con el sonido y la música. Son los sonidos una de las primeras experiencias con el exterior, incluso antes de nacer.

Sin embargo, el contexto en el que un niño crece y desarrolla sus habilidades para la vida, influye de una manera determinante. Cuando hablamos del aprendizaje de la música y la técnica de un instrumento son cruciales las condiciones en las que uno puede acercarse a ello.

En la década de los ochenta, en Hermosillo, eran muy pocos los lugares donde se podía aspirar a aprender el lenguaje de la música.

Siendo un niño tuve la gran oportunidad de conocer a la maestra Emiliana de Zubeldía, pionera de la música de concierto en esta ciudad, que dirigía el coro de la Universidad

\footnotetext{
* Clarinetista de la Orquesta de Baja California, director de la Orquesta Cerro Colorado del programa Redes 2025, profesor de clarinete de la licenciatura de la Facultad de Artes de la Universidad Autónoma de Baja California.
} 


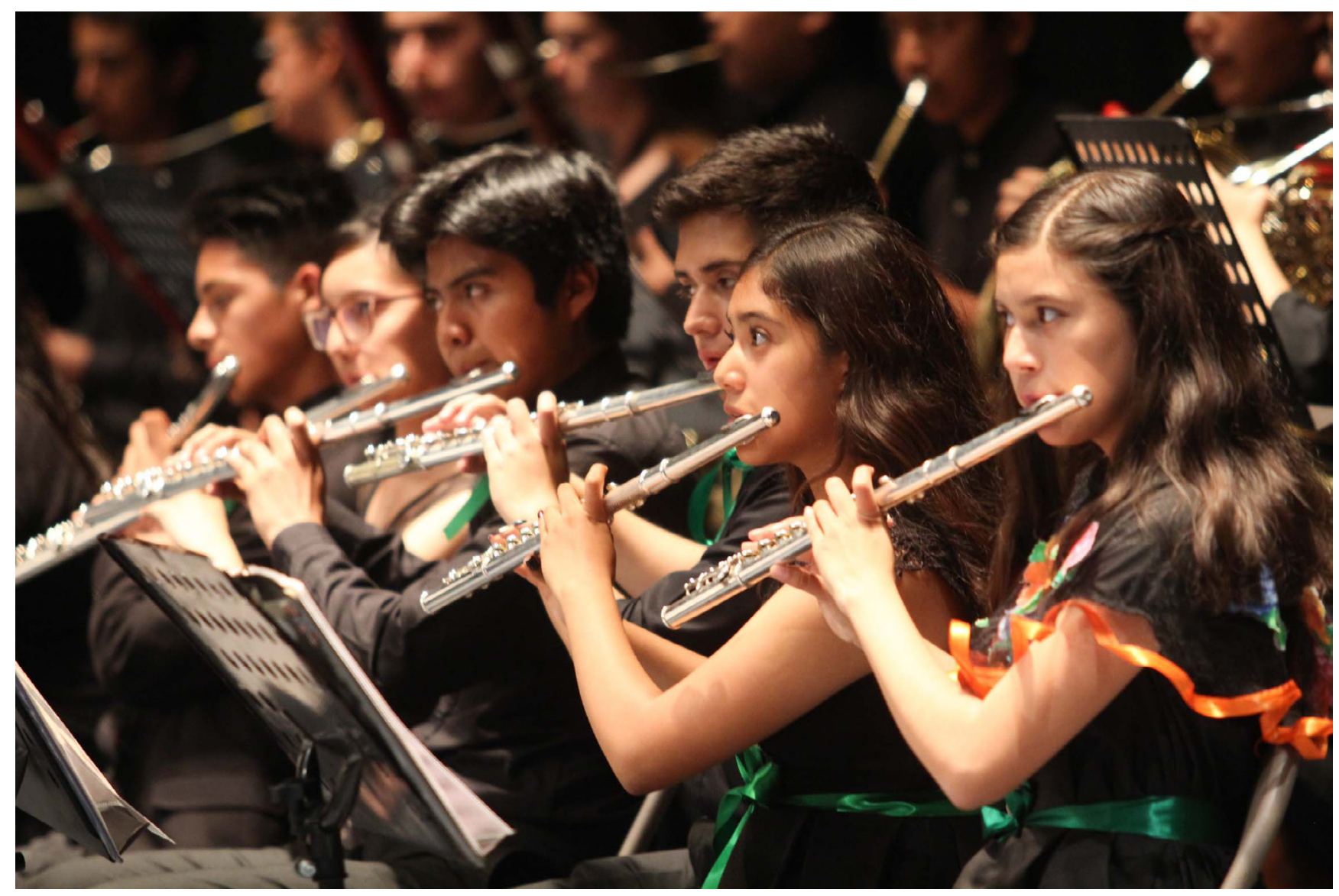

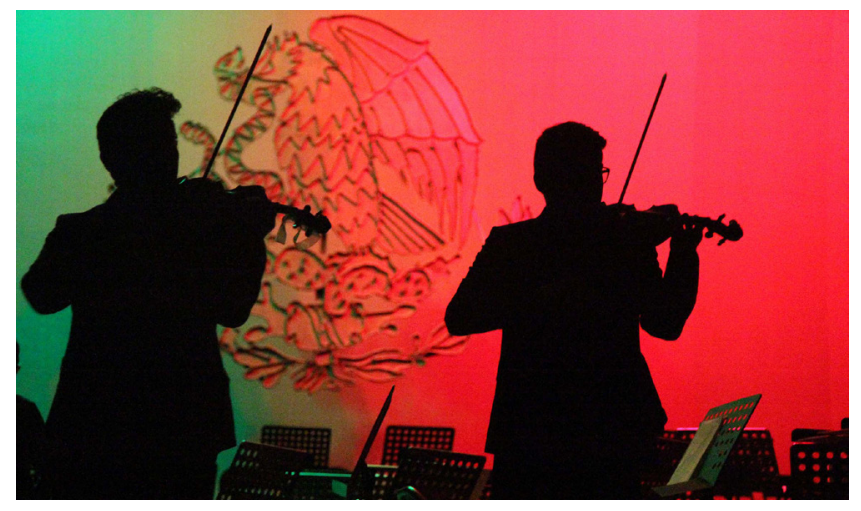

de Sonora (Unison) conformado mayoritariamente por jóvenes y adultos aficionados a la música coral y algunos pianistas.

$\mathrm{Su}$ examen de conocimientos musicales nunca lo olvidaré: repetir ritmos en su escritorio de metal con el puño de la mano y entonar sonidos e intervalos que ella tocaba al piano.

Más adelante, tuve la oportunidad de estudiar música en la Escuela de disciplinas Musicales (EDIMUS), de la maestra Leticia Varela, una excelente pedagoga formada en Alemania que logró sostener por una buena cantidad de años un proyecto independiente por el que pasamos varias generaciones de amantes de la música.

En esta escuela conocí a estupendos maestros, pero las dos más importantes fueron Beatriz Estandarte que puso una flauta dulce en mis manos y Otila Caballero que me abrió los ojos al arte y a la práctica musical.

En esos años, Hermosillo no tenía un lugar dedicado a la enseñanza de la música que tuviera un interés especial por los niños y el desarrollo de sus capacidades. Quizás lo que existía eran esfuerzos aislados y no permanentes que se presentaban como únicas opciones.

Los últimos años que viví en esta calurosa ciudad del noroeste (1986-1988) ingresé a la banda de la Unison con el maestro Horacio Lagarda y ahí pude conocer lo que después se convirtió en mi pasión, mi profesión y mi forma de vida: el clarinete. Además, el contacto con jóvenes universitarios me enseñó prematuramente la vida de los músicos en activo; conocí, tocando, el estado de Sonora y reafirmé lo que sería para siempre mi opción de vida: ser músico.

Después de esta etapa inicial en Sonora, tuve un acercamiento definitivo y profesional viviendo en la Ciudad de México en un lapso de doce años, que me permitió transformarme en un clarinetista y maestro de música. 


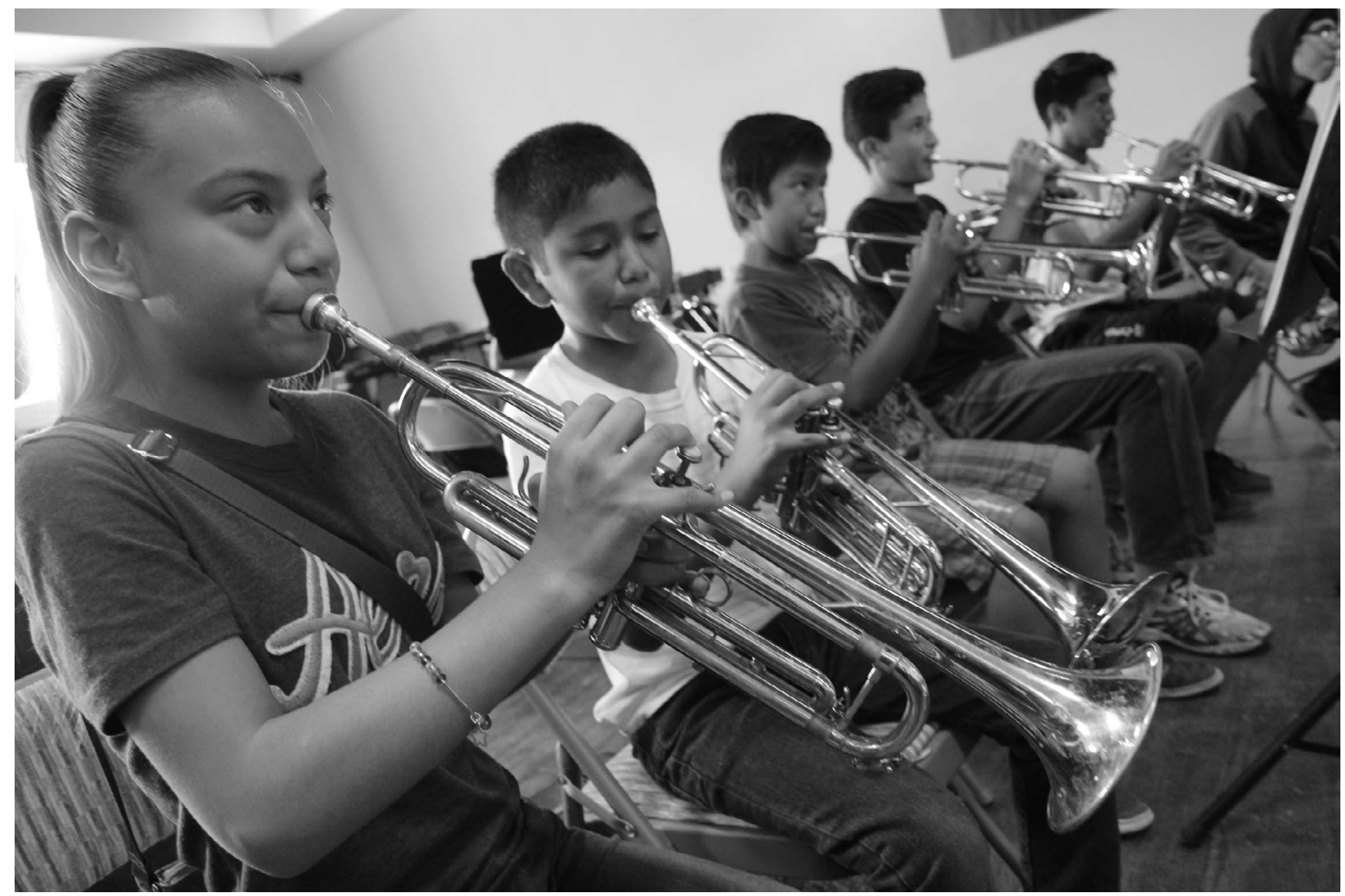

Y así fue como llegué a Tijuana en el año 2000. Llegar a la frontera, además del choque cultural, implicó una nueva adaptación y aún más, llegar a un territorio donde la música abunda en estilos y géneros de muy diversas regiones y países, pero donde la infraestructura cultural es muy incipiente y las opciones de desarrollo en las artes de manera profesional es una realidad que hace pocos años que comenzó a ser viable.

Vivir de músico que se dedica a la música de concierto en un territorio con una historia reciente y población tan heterogénea no es cosa fácil. De modo que, además de aprender a interactuar con los espacios limitados para la práctica profesional de la música, tuve que entender que aquí la labor sería también la de crear públicos y aprender a ser gestor y promotor de mis propias actividades.

Sin embargo, debo reconocer que pertenecer a la Orquesta de Baja California (OBC) me dio una ventaja considerable para poder integrarme en buena medida al ambiente cultural de la región y comenzar, desde ahí, a tener una participación muy activa dentro de él.

Fue en el año 2010, en el seno del Centro de Artes Musicales (CAM), que es la sede de la OBC, cuando se propuso iniciar un proyecto denominado Redes 2025, que tendría como eje el trabajo musical en comunidades alejadas de los centros de cultura.

Este programa de enseñanza musical comunitaria del Centro de Artes Musicales está diseñado para trabajar en torno al concepto de la música como agente de cambio que promueve el sentido de pertenencia entre los niños y jóvenes que la conforman y refuerza los principios de convivencia, tales como el trabajo en equipo (multinivel), participación con la comunidad, solidaridad, compromiso, disciplina y responsabilidad, entre otros.

El diseño académico del programa Redes 2025 estuvo a cargo de los maestros Sergio Ramírez Cárdenas y Eduardo García Barrios, inspirado en el Programa de Orquestas Juveniles de Venezuela y el de los núcleos orquestales de México y la Orquesta Escuela Carlos Chávez.

GLORIA DUARTE

La experiencia académica y los productos artísticos que se desprenden del trabajo cotidiano en comunidades y dentro del CAM con respecto a este programa deberían tener un fundamento esencial: la posibilidad de trabajar con valores como la identidad y el trabajo en equipo a partir de estrategias de trabajo comunitario.

Para los maestros de este programa entender qué es lo comunitario y cómo se puede trabajar en sus diferentes expresiones ha implicado un largo esfuerzo de capacitación, experimentación, implementación de diferentes metodologías y el acompañamiento con especialistas de otras disciplinas, ya sea por alianzas o por apoyo directo a nuestro programa. 
Creemos que las familias de nuestros alumnos y los lugares donde trabajamos son susceptibles de recibir un impulso positivo a partir de nuestro trabajo con la música cuando esta es concebida como una herramienta para el desarrollo de capacidades individuales y colectivas.

En ciudades como Tijuana, la integración de los grupos sociales con carga cultural diferente y la posibilidad de habitar los espacios públicos son situaciones que se presentan como problemáticas que no siempre se toman en cuenta.

El tejido social se fortalece cuando los grupos cuentan con un entorno sin violencia, leyes explicitas y transparentes, servicios básicos, abundantes vías, medios y lenguajes de comunicación y con amplios espacios públicos incluyentes que usan con libertad y frecuencia. También se fortalece cuando los grupos han construido una memoria común, cuando cuentan con recursos naturales, de infraestructura, económicos, científicos y culturales, cuando conocen medios para convertir recursos comunales en bienes públicos, cuando comparten paradigmas consensuados y diseñan proyectos que los acerquen a ellos, y cuando pertenecen a redes que van más allá de la comunidad misma.

JOSÉ CARREÑO CARLÓN
Desde el punto de vista de desarrollo comunitario, el programa Redes 2025 aspira a que el trabajo cotidiano dentro de las estructuras corales y orquestales, así como de ensambles y clases de especialidad instrumental, coadyuven en la creación de espacios donde los jóvenes puedan desarrollar capacidades y tener una experiencia profunda con la música.

Es por ello que se aplica sin costo económico particularmente en colonias de altos índices de violencia y drogadicción como Mariano Matamoros, Camino Verde, La Libertad, Zona Centro, Granjas Familiares, Colonia Chihuahua, entre otros lugares, y en la sede matriz que es el CAM.

Con este trabajo intensivo queremos contribuir a evitar la ruta de acceso de los jóvenes a situaciones de mayor marginación y a ser presa inmediata de los cárteles y los grupos delincuenciales. Asimismo, se les aporta un oficio que al cabo de un esfuerzo sostenido por años los puede hacer muy buenos candidatos para la profesionalización y su incorporación al mercado laboral, como ya hemos visto en muchos casos.

Es así que podemos decir con certeza que "Redes 2025 durante siete años y medio, ha logrado transformar la vida interna de cientos de familias que se han involucrado de manera decidida" con uno o más niños inscritos

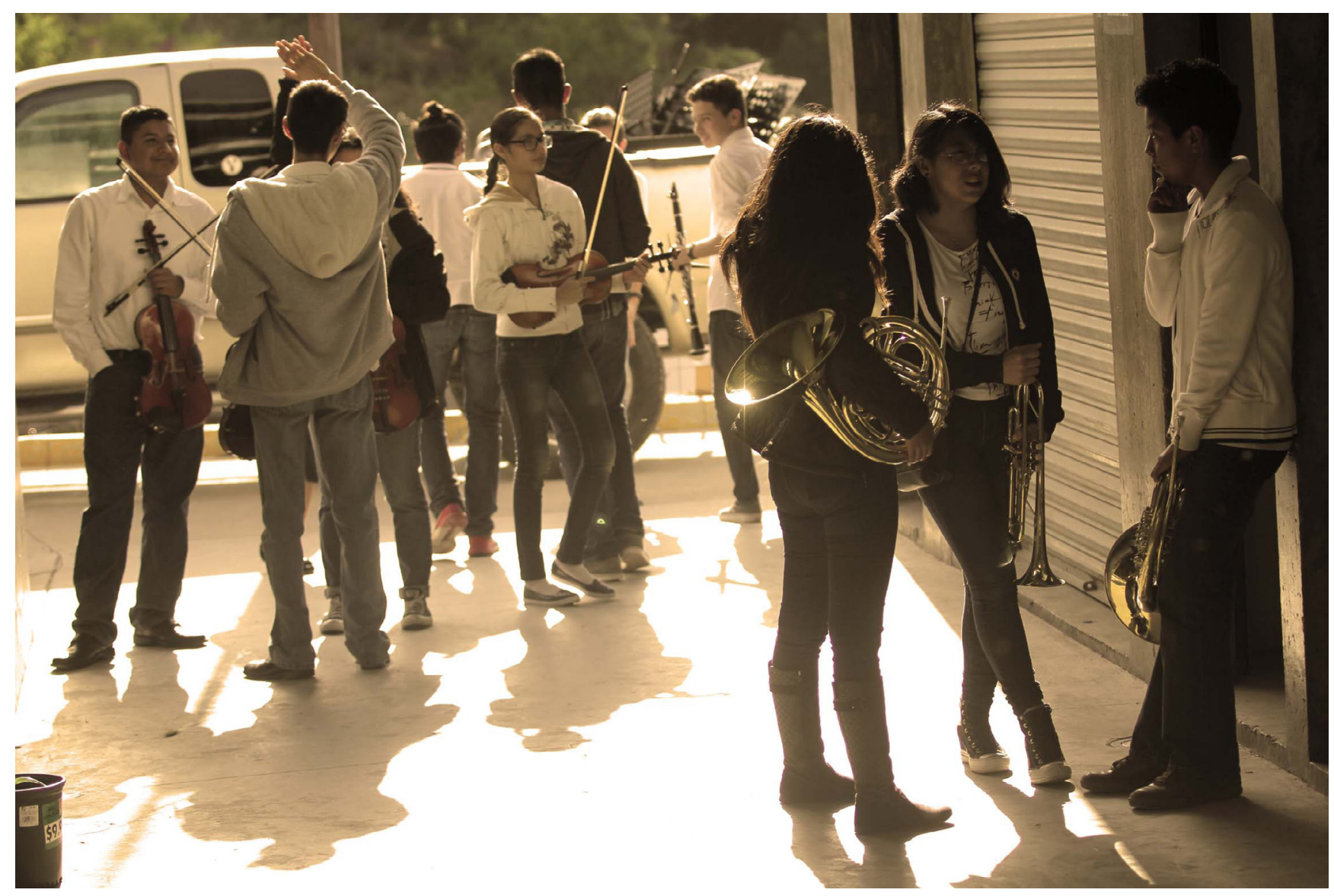


simultáneamente al programa con participaciones de uno a más de siete años constantes.

Este programa para su supervivencia cuenta con el apoyo del CAM y de la Fundación de las Artes Musicales para la obtención de los recursos como pago a docentes, instrumentos, espacios de trabajo, vinculación con instituciones y proyectos de diferente naturaleza.

En siete años se han realizado viajes de niños a la Ciudad de México a la Orquesta Infantil de México; giras regionales numerosas ocasiones, a California de manera constante; Idaho y Nueva York en Estados Unidos, Colombia, Italia en diferentes ocasiones; Francia, Holanda y España, entre otros lugares.

Tenemos exalumnos estudiando la carrera profesional de música en la Facultad de Artes de la UABC, en la Orquesta Escuela Carlos Chávez de la ciudad de México y en el Conservatorio de las Rosas de Morelia.

Asimismo, exalumnos que estudian otras carreras han sido contratados por el programa Redes como maestros en los centros comunitarios y las clases dentro del CAM.

De manera periódica los participantes del programa son invitados a realizar audiciones para participar en conciertos especiales con la OBC y otras orquestas como Mainly
Mozart de San Diego, Sinfónica Juvenil de Tijuana, la Fundación Fronteras Abiertas de Italia, etc.

Uno de los objetivos académicos ha sido la posibilidad de incorporar alumnos egresados de Redes 2025 al propedéutico y licenciaturas que comenzarán a ofertarse con aprobación oficial en un par de años más. De esta forma se garantizaría la necesidad de maestros de música con perfil comunitario y nuevos integrantes de excelente nivel a la Orquesta de Baja California.

Por último, las alianzas de Redes 2025 lo hacen un programa exitoso y multidisciplinario en el que se comparten experiencias y conocimientos. Maestros como Eduardo García Barrios, Sergio Ramírez Cárdenas de México, Susan Siman de Venezuela, Francesco Grigolo de Italia, maestros de Nueva York, de San Diego, de Holanda, de Argentina, de la Ciudad de México y los mejores maestros del estado de Baja California, incluyendo a integrantes de la OBC, hemos tenido una participación importante a lo largo de estos años dentro del programa Redes.

Además, nos hemos vinculado a investigaciones y proyectos con la UNY en un programa de enseñanza de las matemáticas y la música, producción de óperas con la fundación Fronteras Abiertas de Italia, producción de conciertos con la OBC, con la fundación Simanoff de Miami,

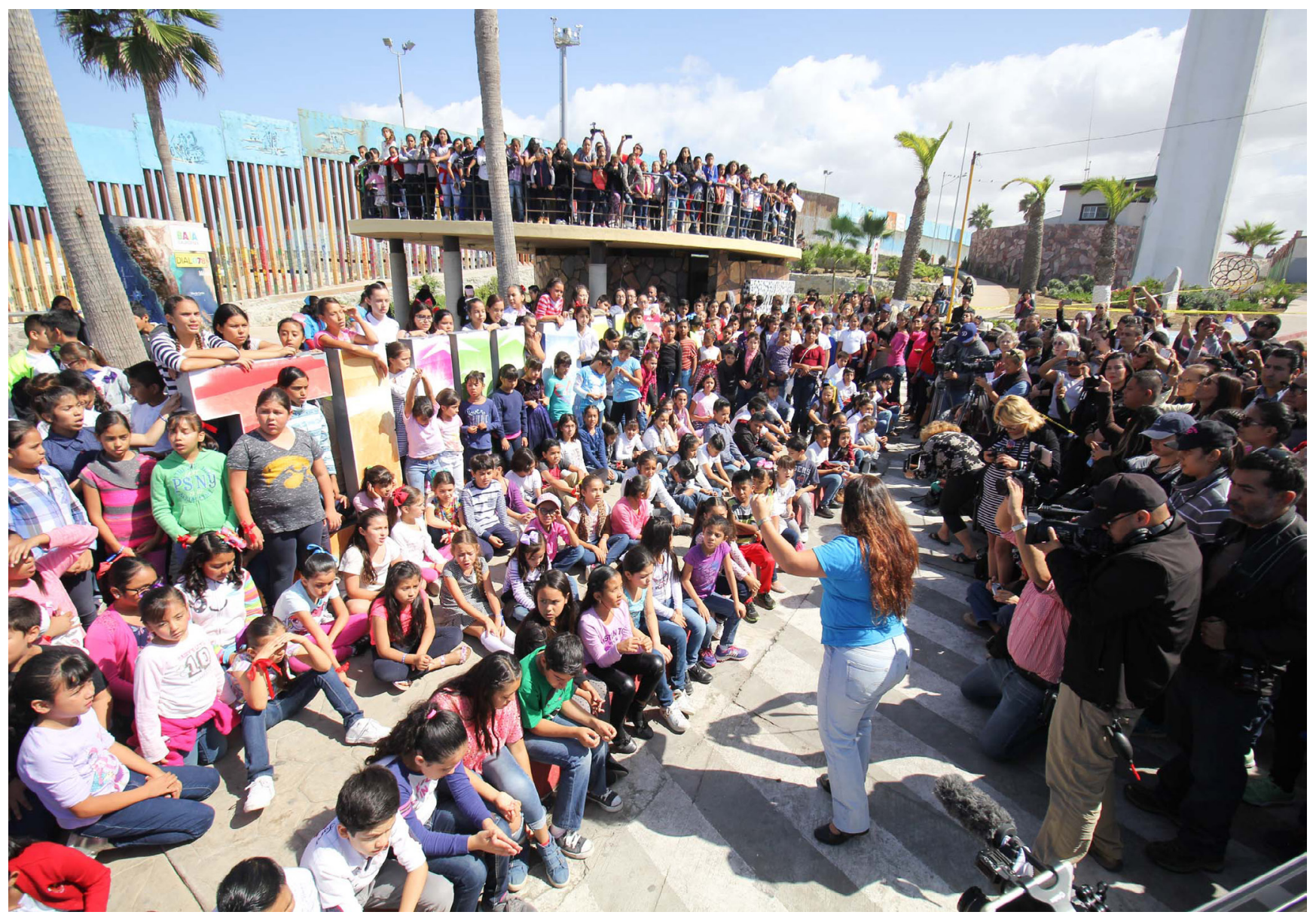




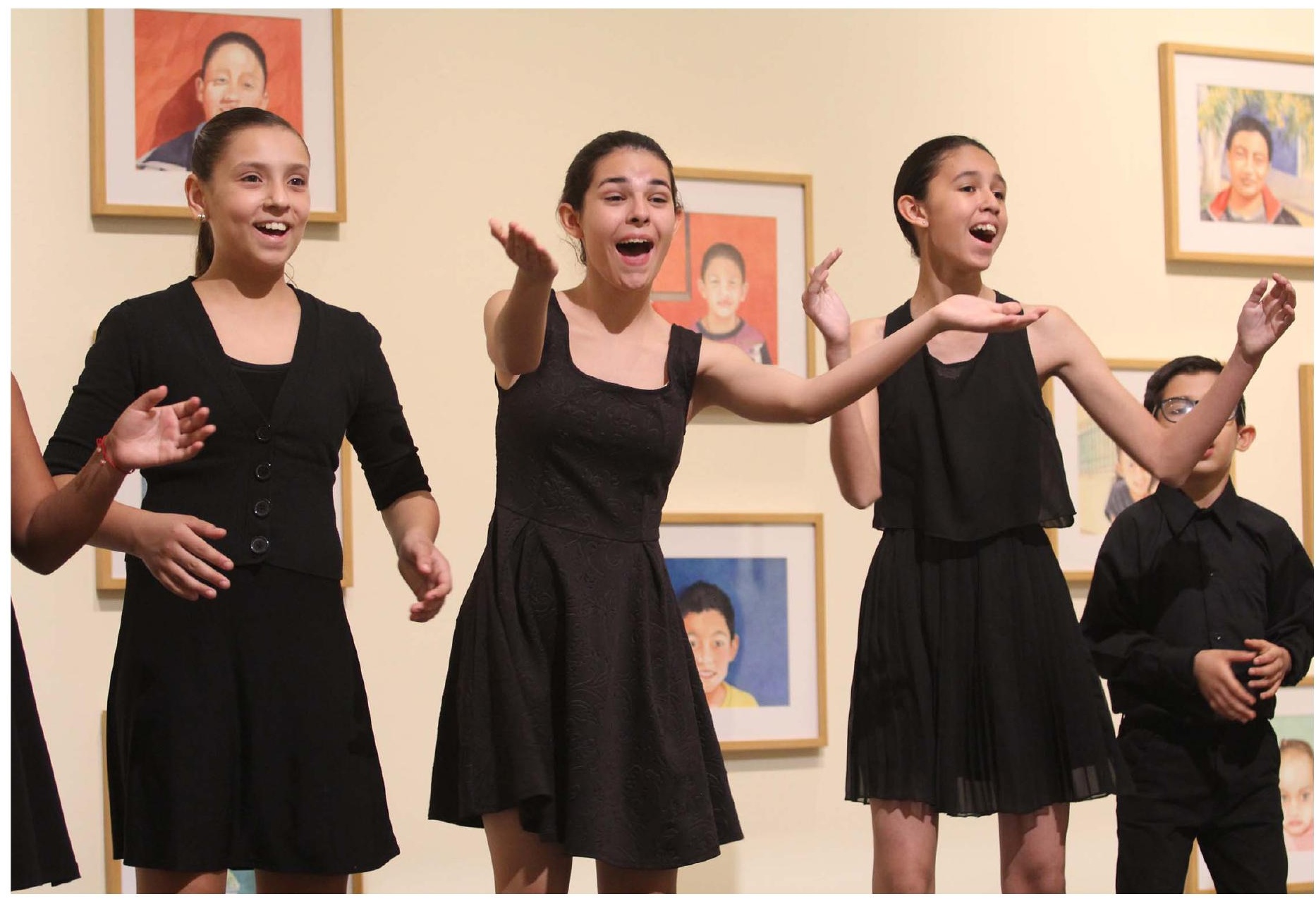

la orquesta juvenil Mainly Mozart y su programa en San Diego, los coros de Promotora de Bellas Artes de Tijuana y la asociación civil Ópera de Tijuana y más recientemente con la organización internacional Playing for Change.

Después de ocho años de tener una participación muy activa mi mirada como músico profesional y como docente de la música ha cambiado, de una visión en la que se prepara a una élite de alumnos privilegiados y con predisposición a la música por la del trabajo colectivo con aspectos comunitarios en el que todos son bienvenidos; cada quien aporta una parte y entre todos construimos un espacio de desarrollo humano con la música como un lenguaje en común.

Mi origen musical y el desarrollo profesional que he encontrado ahora tienen un vínculo: el deseo enorme de transformar nuestra realidad hacia espacios donde el sonido de la orquesta y los coros nos sostienen y nos dan el aliento necesario para lograrlo.

Mi gran aprendizaje ha sido que en principio lo que se necesita es querer trabajar colectivamente en torno a lo que pueda ser lo más difícil, con herramientas universales como el lenguaje de la música.
Creo que hoy más que nunca, el arte juega un papel fundamental en el desarrollo de las comunidades y ya no solo como producto cultural o de disfrute estético.

Y si ese esfuerzo se realiza con disciplinas como la sociología, la psicología y el trabajo social, su poder de transformación comunitaria se potencializa y mejora sustancialmente.

El futuro del arte, creo, debe ser alentador y propositivo en nuestras comunidades.

\section{Referencias}

Torres Parès, P. (2003). El arte de educar. Una concepción integradora de la educación musical. México: Santillana.

Duarte, G. (2017). Cuaderno para pensar y decidir del Centro de Artes Musicales $N^{\circ}$ 2. Tijuana, BC: UAMUS.

Carreño Carlón, J. (2017). Cultura de paz, palabra y memoria. Un modelo de gestión cultural comunitario en Apatzingán, Michoacán. Ciudad de México: Fondo de Cultura Económica. 